\title{
Forschung an österreichischen Fachhochschulen
}

\author{
P. Rössler OVE, IEEE
}

Online publiziert am 16. Dezember 2019

(C) Springer-Verlag GmbH Austria, ein Teil von Springer Nature 2019

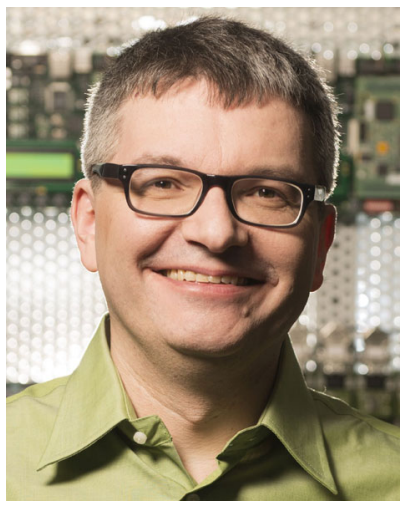

FH-Prof. Dipl.-Ing. Dr. techn. Peter Rössler
Liebe Leserinnen und Leser,

„Forschung an Fachhochschulen, das gibt es?" Solche oder ähnliche Aussagen bekam ich zu hören, als ich im Jahr 2005 nach über acht Jahren beruflicher Tätigkeit als Universitäts- und Projektassistent von der Technischen Universität Wien an die FH Technikum Wien wechselte. Etwas naiv dachte ich zunächst, meine F\&E-Tätigkeiten einfach nahtlos an der Fachhochschule fortsetzen zu können, bis mir alsbald klar wurde, dass die Systeme an österreichischen Universitäten und Fachhochschulen diesbezüglich doch einige markante Unterschiede und Eigenheiten aufweisen. Persönlich fühlte ich mich damals als eine in der Forschung tätige Person an der $\mathrm{FH}$ eher als Exot und hatte manchmal den Eindruck, von einigen Kollegen hinsichtlich meiner F\&E-Agenden vor allem als jemand gesehen zu werden, der Geld kostet - nämlich Eigenmittel der Fachhochschule. Zwar hat sich die Situation der Forschung an österreichischen Fachhochschulen nach meiner Beobachtung seither etwas verändert, doch einige spezielle Rahmenbedingungen sind geblieben, auf die es sich lohnt, eingangs einen kurzen Blick zu werfen (weitere Details finden sich, ebenfalls in diesem Heft, in einem ausführlichen Übersichtsbeitrag von Kollegin Giuliana Sabbatini und Kollege Johann Kastner).

Zunächst ist es interessant festzustellen, dass Fachhochschulen in Österreich über das Bundesgesetz für Fachhochschul-Studiengänge - FHStG zwar einen gesetzlich verankerten Auftrag zur Forschung haben, die finanziellen Mittel des Bundes jedoch ausschließlich zum Zwecke der Lehre sowie dafür notwendiger Administration und Infrastruktur verwendet werden dürfen. Forschung an $\mathrm{FHs}$ muss damit anders finanziert werden, zum Beispiel durch nationale oder internationale Fördermittel (über gemeinhin bekannte Institutionen wie etwa FFG, CDG und FWF) oder etwa durch direkte Beauftragung der Industrie. In ersterem Fall befindet man sich in direktem Wettbewerb mit Universitäten und außeruniversitären Forschungseinrichtungen, was gerade das Hochstarten von neuen Themenfeldern zu einer nicht leicht zu meisternden Hürde macht, zumal die zeitlichen Ressourcen durch Tätigkeiten in Lehre und Administration an der Fachhochschule oft beschränkt sind. Auftragsforschung durch die Industrie erscheint im Kontext von F\&E hingegen logisch, gerade wegen der Ausrichtung von FHs auf anwendungsnahe Forschung (also mit typischerweise kürzerem Zeithorizont zu einem Produkt, als dies bei der grundlagenorientierteren Forschung an Universitäten der Fall ist - Ausnahmen bestätigen die Regel). Problematisch ist hier allerdings wiederum die oftmalige Beschränkung der Publikationstätigkeit aus Gründen der Vertraulichkeit und Geheimhaltung oder die Gefahr, dass diese Art der Beauftragung in eine reine Produktentwicklung mündet - mit überschaubarem Gewinn von neuen Erkenntnissen.

"Spannend" ist es andererseits hingegen immer, nach Beendigung eines mehrjährigen F\&E-Projekts eine zeitnahe Anschlussfinanzierung zu finden. Gelingt dies über einen längeren Zeitraum nicht und wandert Personal und damit Kompetenzen vom Standort ab, muss man, salopp gesagt, mit dem Strukturaufbau wieder von vorne beginnen. Derartige Herausforderungen in der Forschung sind auch Universitäten und außeruniversitären Forschungsinstitutionen freilich wohlbekannt, an FHs sind diese regelmäßigen Problemstellungen wegen der fehlenden Basisfinanzierung des Bundes für F\&E vielleicht aber noch ein wenig herausfordernder.

Sieht man sich das Verhältnis zwischen Forschung und Lehre an österreichischen Fachhochschulen an, ist klar festzustellen, dass die Lehrtätigkeit hier bei weitem dominiert. Letztere Aufgabe erfüllen FHs aus meiner Sicht äußerst lobenswert, was sicherlich auch wiederum systemimmanent bzw. Resultat der speziellen Rahmenbedingungen ist (beispielsweise ein garantiertes Betreuungsverhältnis durch eine Studienplatzfinanzierung). Lehre ist also das Kerngeschäft der Fachhochschulen, wie es oft formuliert wird. Zu meiner aktiven Zeit als Assistent an der TU Wien gab es im Gegensatz dazu kaum Kolleginnen oder Kollegen, die sich exklusiv der Lehre oder ausschließlich F\&E widmeten. Auch war es üblich, dass man bereits als Diplomand an aktuellen Forschungsthemen mitarbeitete, unter Anleitung und Mitwirkung eines Assistenten oder Professors wissenschaftlich publizierte und zu einem späteren Zeitpunkt, wenn man nach Abschluss des Studiums dann vielleicht als junger Projektassistent an der Universität arbeitete, Einblicke in F\&EProjektmanagement und in die Erstellung von neuen Projektanträgen erhielt. Diese Tradition, nämlich den Nachwuchs damit automatisch an die Forschung heranzuführen, ist hingegen an Fachhochschulen typischerweise weniger verbreitet. Einer der Gründe dafür ist sicher, dass die Mittel des Bundes, wie bereits erwähnt, exklusiv für die Lehre zur Verfügung stehen. Zudem existiert die rechtliche Basis für Fachhochschulen in Österreich erst seit 1993, und so war man in der Anfangsphase vor allem einmal damit beschäftigt, den Studienbetrieb an den Standorten aufzubauen und dachte dabei zunächst wohl weniger an Forschung.

An dieser Stelle darf auch gleich die provokante Zwischenfrage gestellt werden, ob es denn Forschung an Fachhochschulen überhaupt braucht? Oder, wie man es zuweilen auch etwas anders formuliert hört: Kann man Forschung an Hochschulen nicht gänzlich

Rössler, Peter, Department Electronics Engineering, FH Technikum Wien, Höchstädtplatz 6, 1200 Wien, Österreich (E-Mail: peter.roessler@technikum-wien.at) 
den Universitäten überlassen? Für mich persönlich ist die Antwort auf derartige Fragen klar: Ich halte F\&E, auch an Fachhochschulen, als essentiell notwendig für den Aufbau, den Erhalt und die Weiterentwicklung von Kompetenzen am Standort! Zudem ist es für das akademische Personal wohl spannender und motivierender, nicht nur Wissen zu vermitteln, sondern im Rahmen von Forschungsprojekten auch selbst neues Wissen zu generieren. Dies gilt natürlich auch im Kontext einer Steigerung von Aktualität und Attraktivität der Lehre, indem Studierenden z.B. Projekt-, Bachelor- oder Masterarbeiten in thematischer Anknüpfung an aktuelle F\&E-Projekte angeboten werden können. Schließlich leisten FHs für österreichische Unternehmen unbestreitbar einen wertvollen Beitrag, indem sie beispielsweise neue Ideen und Technologien, im Rahmen einer anwendungsnahen F\&E, also über einen Proof-of-Concept hinaus, für einen Einsatz in Produkten und damit auch industriell nutzbar machen.

Obwohl sich Forschung an Fachhochschulen sicherlich nicht so dominierend wie an Universitäten oder außeruniversitären Forschungseinrichtungen darstellt, können österreichische FHs in den nunmehr 25 Jahren ihres Bestands beachtliche Erfolge vorweisen. Ziel dieses Schwerpunktheftes ist es, im Rahmen von acht Beiträgen einen Blick auf die F\&E-Landschaft an österreichischen Fachhochschulen im Bereich der Elektrotechnik und Informationstechnik zu werfen.

Im ersten Artikel führen Giuliana Sabbatini und Johann Kastner, Mitglied bzw. Vorsitzender des F\&E-Ausschusses der österreichischen Fachhochschulkonferenz (FHK), mit einem umfassenden Übersichtsbeitrag über die Forschungs- und Entwicklungsleistung der österreichischen Fachhochschulen aus sektorinterner Sicht, in die Thematik ein. Der nächste Beitrag von der FH Joanneum beschreibt eindrucksvoll die langjährigen und vielfältigen Aktivitäten des Instituts für Electronic Engineering in Bereichen wie Leistungselektronik, Model-Based Realtime Computing oder Elektromagnetischer Verträglichkeit. Danach zeigt die FH Kärnten in ihrem Beitrag, wie interdisziplinäre Forschung zwischen Thematiken wie Health \& Assisted Technologies und Mikroelektronik erfolgreich funktionieren kann.

Gleich drei Artikel kommen aus dem Bereich der Energietechnik: Vom Standort Wels der FH Oberösterreich wird über die Entwicklung eines Mittelspannungs-Batteriespeicherkonzepts berichtet, während die FH Vorarlberg einen Überblick über ihre Forschungsarbeiten im Kontext Autonomous Demand Side Management in Stromnetzen gibt. Die FH Salzburg schlägt in ihrem Artikel über Security- und Privacy-Aspekte in Smart Grids eine thematische Brücke zur Infor- matik. Aus ebendieser Disziplin, nämlich der Informatik, stammt ein weiterer Beitrag der FH St. Pölten, der sich mit Angriffen auf Computersysteme, genauer gesagt mit der Erkennung und Interpretation von anomalem Verhalten in Systemprozessen beschäftigt. Zuletzt beschreibt ein Artikel der FH Technikum Wien aktuelle Trends und Forschungsfragen im Bereich der programmierbaren Logikbausteine, die sich seit ihrer Markteinführung mittlerweile zu komplexen Multiprozessor-Systems-on-Chip entwickelt haben und damit als Schlüsselkomponenten für Electronic Based Systems in einer Vielzahl von Applikationsdomänen gesehen werden können. Anzumerken sei abschließend, dass fünf der sieben technischen Beiträge an Forschungsaktivitäten in Josef-Ressel-Zentren anknüpfen, die ja als Exzellenzzentren an Fachhochschulen positioniert wurden. Außerdem soll noch darauf hingewiesen werden, dass hier aus Platzgründen natürlich nur ein exemplarischer Überblick im Bereich von F\&E an Fachhochschulen gegeben werden kann und selbstverständlich auch an anderen FH-Standorten in Österreich (siehe etwa Webseite der FHK ${ }^{1}$ ) Forschung betrieben wird.

Wenn ich mich nun am Ende dieser Einleitung zu einem etwas pathetischen Schlusswort hinreißen lasse, kann ich aufgrund meiner langjährigen Beschäftigung mit F\&E nicht anders, als den unterschiedlichen Stellenwert von Forschung an Fachhochschulen und Universitäten oder außeruniversitären Forschungseinrichtungen etwas ernüchtert zur Kenntnis zu nehmen. Ich hoffe aber, dass dieses Schwerpunktheft zeigt, dass es auch an österreichischen Fachhochschulen eine Reihe von findigen Köpfen gibt, die mit ihren Ideen, F\&E-Arbeiten und vor allem mit ihrer Begeisterung und Motivation permanent neues Land betreten und damit - trotz nicht einfacher Rahmenbedingungen - ihren Beitrag zur Weiterentwicklung von Wissenschaft, Wirtschaft und der Gesellschaft leisten. Es sei dies letztendlich auch ein Aufruf an Entscheidungsträger und die Politik, das Kreativpotential im Bereich der Forschung (egal ob an Fachhochschulen, Universitäten oder außeruniversitären Forschungsinstitutionen!) bestmöglichst zu unterstützen und für die geeigneten Rahmenbedingungen zu sorgen.

Ich darf mich abschließend noch bei allen Autorinnen und Autoren der vorliegenden Arbeiten bedanken und Ihnen, liebe Leserinnen und Leser, viel Vergnügen beim Lesen der Beiträge wünschen!

Hinweis des Verlags Der Verlag bleibt in Hinblick auf geografische Zuordnungen und Gebietsbezeichnungen in veröffentlichten Karten und Institutsadressen neutral.

\footnotetext{
${ }^{1}$ http://www.fhk.ac.at.
} 